\title{
Polish Medical Society of Radiology and Polish Society of Rheumatology recommendations for magnetic resonance imaging of musculoskeletal disorders in rheumatology*
}

\author{
Iwona Sudoł-Szopińska ${ }^{1 *},{ }^{* *}$, Andrzej Urbanik ${ }^{2 *}$, Wadim Wojciechowski ${ }^{2 *}$, Agnieszka Warczyńska ${ }^{1 *}$, \\ Katarzyna Kapuścińska ${ }^{2 *}$, Mariusz Korkosz ${ }^{3 * *}$, Stawomir Jeka ${ }^{4 * *}$, Brygida Kwiatkowska ${ }^{5 * *}$ \\ ${ }^{1}$ Department of Radiology, E. Reicher Institute of Rheumatology, Warsaw, Poland \\ ${ }^{2}$ Chair of Radiology, Jagiellonian University Medical College, Cracow, Poland \\ ${ }^{3}$ Department of Rheumatology, Clinic of Internal Medicine, University Hospital, Cracow, Poland \\ ${ }^{4}$ Clinic of Rheumatology and Systemic Connective Tissue Disorders, J. Biziel University Hospital No. 2, Bydgoszcz, Poland \\ ${ }^{5}$ Early Arthritis Clinic, E. Reicher Institute of Rheumatology, Warsaw, Poland \\ * Polish Medical Society of Radiology, ${ }^{* *}$ Polish Society of Rheumatology
}

\begin{abstract}
This document presents the recommendations of the Polish Medical Society of Radiology (PLTR) and the Polish Society of Polish Society of Rheumatology (PTR) regarding the standards of collaboration between radiologists and rheumatologists so as to optimize the diagnostics and treatment of patients with rheumatic diseases of the musculoskeletal system, including rheumatoid arthritis and spondyloarthropathies.
\end{abstract}

Key words: magnetic resonance imaging, radiologic health, radiology information systems.

\section{Background}

This document presents the recommendations of the Polish Medical Society of Radiology (PLTR) and the Polish Society of Rheumatology (PTR) regarding the standards of collaboration between radiologists and rheumatologists in order to standardize the diagnostic procedure and treatment of patients with rheumatic diseases of the musculoskeletal system, including rheumatoid arthritis and spondyloarthropathies.

The document has been prepared on the basis of local clinical expertise as well as the guidelines of the European Society of Skeletal Radiology (ESSR), the Assessment of SpondyloArthritis International Society (ASAS) and European League Against Rheumatism (EULAR) [1-9].

Following issues are discussed in the recommendations:

- the role of MRI in the current criteria for classification of rheumatic diseases;
- MRI referrals;

- the range of MRI scans;

- MRI protocols;

- MRI interpretation;

- the role of MRI in treatment.

\section{The role of MRI in the criteria for classification of rheumatic diseases}

In clinical practice, MRI of musculoskeletal system is performed in patients with rheumatic diseases to diagnose inflammation within peripheral joints, tendon sheaths, bursae, the spine, entheses of the ligaments, joint capsule and fascia as well as complications of these conditions.

Most commonly, MRI is performed in differential diagnosis of:

\footnotetext{
*This paper was published in Pol J Radiol 2015; 80: 259-265. DOI: 10.12659/PJR.893670

Address for correspondence:

Iwona Sudoł-Szopińska, Department of Radiology, E. Reicher Institute of Rheumatology, Spartańska 1 Str., 02-637 Warsaw, Poland, e-mail: sudolszopinska@gmail.com
} 
1. Spondyloarthropathies (SpA; spondyloarthritis; inflammation of the spine accompanied by inflammation of peripheral joints), including:

a. Axial spondyloarthropathy: inflammation of the sacroiliac joints (sacroiliitis) and the spine (spondylitis);

b. Peripheral spondyloarthropathy: inflammation of the peripheral joints (arthritis), tendon sheaths (tenosynovitis; tendovaginitis) and entheses (enthesitis).

2. Rheumatoid arthritis (RA): inflammations within peripheral joints, tendon sheaths, bursae (bursitis) and the spine;

3. Juvenile idiopathic arthritis (JIA): inflammation within peripheral joints (including temporo-manidibular where MRI is the method of choice), tendon sheats;

4. Any rheumatic diseases involving musculoskeletal system without diagnosis established on the basis of radiographic or ultrasonographic findings: peripheral joints, tendon sheaths, bursae, the spine and muscles.

Main indications for MRI in patients with rheumatic diseases include:

- Assessment of inflammatory lesions, particularly early stages involving joint cavities (synovial thickening, synovial enhancement following contrast agent administration, effusion, bone marrow edema, erosions, inflammatory cysts);

- Assessment of inflammatory lesions in tendon sheaths, tendons and their entheses;

- Assessment of muscles' inflammation (myositis);

- Assessment of complications of rheumatic disorders, e.g cervical spine lesions in the course of RA.

Table I. ASAS 2010 criteria for classification of axial spondyloarthritis [2, 3, 9]

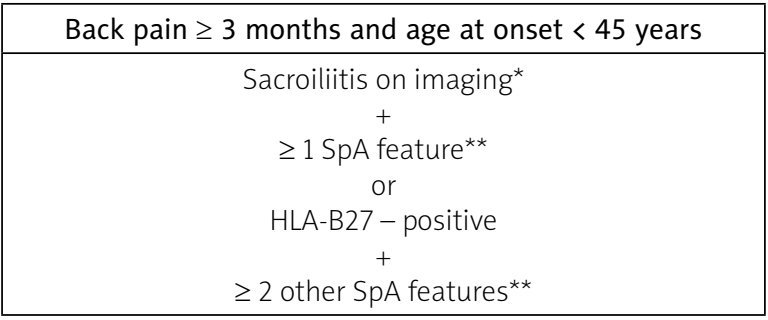

* Active (acute) inflammation on MRI or definite radiographic sacroiliitis according to the modified New York criteria (1984) are highly suggestive of sacroiliitis associated with SpA

** SpA features: inflammatory back pain (at least 4 of the following symptoms must occur: 1. pain onset before the age of 40; 2. insidious onset; 3. improvement with exercise; 4. no improvement with rest; 5. pain at night, with improvement upon getting up), peripheral arthritis, enthesitis, uveitis, dactylitis, psoriasis, Crohn's disease (CD) or ulcerative colitis (UC), good response to non-steroidal antiinflammatory drugs, family history of SPA, HLA$-B 27$, elevated CRP
The advantages of MRI are:

- Ability to assess all articular surfaces;

- Ability to assess inflamed bone marrow (bone marrow edema cannot be detected by any other modality; it may precede articular cartilage destruction);

- Ability to assess the spine and the spinal cord (e.g. spinal stenosis, spondylodiscitis, atlantooccipital alterations along with the assessment of the activity of inflammation);

- Higher specificity due to contrast-enhanced scans;

- Semiquantitative and quantitative inflammation assessment by means of the intensity and extent of contrast enhancement analysis.

\section{MRI in the classification criteria}

\section{Axial spondyloarthropathy}

MRI of sacroiliac joints is most frequently performed in patients with chronic back pain (Table I) whose radiographs do not fulfill the modified New York criteria (Table II).

MRI is most commonly performed to [7]:

- Visualize inflammatory lesions in sacroiliac joints, particularly in young patients or patients with short history of the disease, if clinical and radiological findings are insufficient to confirm the clinical suspicion of the disease;

- Monitor the activity of the disease, with frequency of repeated MRI depending on clinical presentation;

- Monitor structural (chronic) changes of sacroiliac joints and the spine, considering radiography as a modality of choice.

\section{Peripheral spondyloathropathy}

Patients presenting clinical symptoms of peripheral arthritis, enthesitis, or edema of the finger(s) are referred for MRI, as X-ray and ultrasound findings are not diagnostic or normal (Table III), in order to determine the spectrum and activity of inflammatory lesions [7].

Table II. Modified New York criteria for ankylosing spondylitis (1984) [9]

\begin{tabular}{|l|}
\hline \multicolumn{1}{|c|}{ Clinical criteria } \\
\hline - Low back pain for more than 3 months that improves with \\
exercise, but is not released by rest \\
- Limitation of motion of the lumbar spine in the sagittal \\
and frontal planes \\
- Reduced chest expansion (relative to normal values corre- \\
lated for age and sex) \\
$\qquad$ Radiological criterion \\
\hline - Definite radiological lesions, i.e. sacroiliitis grade 2-4 \\
biliterally or grade $\geq 3$ unilaterally
\end{tabular}


Table III. ASAS classification criteria for peripheral spondyloarthritis [3, 5]

\begin{tabular}{|c|c|}
\hline \multicolumn{2}{|c|}{$\begin{array}{c}\text { Peripheral arthritis } \\
\text { (usually asymmetric inflammation of joints within lower extremities, enthesitis, dactylitis - "sausage fingers") } \\
\text { plus } \geq 1 \text { of SpA symptoms or } \geq 2 \text { other SpA symptoms }\end{array}$} \\
\hline $\begin{array}{l}\text { - Psoriasis } \\
\text { - CD or UC } \\
\text { - Preceding infection } \\
\text { - HLA-B27 } \\
\text { - Uveitis } \\
\text { - Sacroiliitis on X-ray or MRI }\end{array}$ & $\begin{array}{l}\text { - Arthritis } \geq 2 \text { other SpA features: } \\
\text { - Enthesitis } \\
\text { - Dactylitis/sausage-like toe or digit } \\
\text { - Inflammatory back pain } \\
\text { - Family history of SpA }\end{array}$ \\
\hline
\end{tabular}

\section{Rheumatoid arthritis}

Imaging studies are not included in current ACR/ EULAR criteria for diagnosing RA (2010). MRI is recommended in case of diagnostic uncertainties when $\mathrm{X}$-ray and ultrasound findings are inconclusive [8]. MRI allows detection of inflammatory changes in joint cavities, tendon sheaths and bursae. MRI is the only imaging modality to evaluate bone marrow edema, which together with the synovium lining joint cavities, sheaths and bursae as well as the intra- and extraarticular adipose tissue, may be an area of active inflammation in the course of RA.

In addition, ACR/EULAR criteria recommend MRI to [8]:

- determine the prognosis (bone marrow edema and synovitis are risk factors for the progression of structural changes);

- predict the treatment responses;

- monitor the disease activity and progression;

- assess the activity of inflammatory lesions and atlantooccipital alterations;

- assess inflammatory lesions not detected in the clinical examination.

\section{Juvenile idiopathic arthritis}

$M R I$ is useful for detection and evaluation of the activity of inflammation in joint cavities, tendon sheaths and bursae, as well as for the monitoring of treatment and complications recognition [10].

\section{MRI referrals}

Based on the clinical examination, the clinician determines the anatomical region to be included in the MRI examination.

The referral should include the following:

- The purpose and the rationale for the examination;

- Suspected diagnosis;

- Information required for the proper conduct of a radiological medical procedure (including symptoms and physical examination findings);
- Results of previous examinations, mainly imaging scans;

- Information on current treatment, its efficacy, and biological treatment if planned;

- Medical history (diseases, injuries, surgeries, particularly of the examined area);

- An information if the comparison with the previous scan is required.

Previous MRI results, X-ray and ultrasound scans of the examined anatomical region should be delivered.

In case of patients with implanted foreign bodies including prosthetic implants, the referring physician should ask the patient contraindications for magnetic resonance examination. Foreign bodies consisting ferromagnetic materials are a contraindication for MRI generating strong magnetic field. If indicated, the examination may be performed using a low field scanner.

Intravenous contrast agent is frequently administered. Due to the risk of nephrogenic systemic fibrosis (NSF) following the administration of gadolinium in patients with renal failure (the risk varies for different contrast agents and the stage of renal failure), patients should provide the plasma creatinine level or glomerular filtration rate (GFR).

The contrast agent is administered on the approval of the radiologist, based on the referral data and the overview of the unenhanced scan.

\section{The range of MRI scans}

According to the referral, examination of specific anatomical regions is performed, using special spinal or body phased-array coils.

If indicated, whole body MRI scans are also acquired in search for active inflammation.

When referring patients to peripheral joint imaging using a low field scanner dedicated for examination of small joints, one should consider its limited field of view of around $12 \mathrm{~cm}$. 
For axial SpA, the Polish Medical Society of Radiology recommends whole-spine MRI. When examining hands, a scan of the opposite side should also frequently be acquired.

\section{MRI protocols}

MRI protocols can be adjusted according to local experience, taking into consideration the scanner specifications, available coils and image quality in particular sequences. TIRM/STIR, T2 FS, and PD FS sequences may be used interchangea.

The size of the field of view and the matrix as well as the slice thickness should be appropriately adjusted so as to ensure maximum image quality within reasonable examination times. The recommended slice thickness is $3 \mathrm{~mm}$, with interslice gap of $0.3 \mathrm{~mm}$.

Contrast agent is administered intravenously if indicated.

Listed below are MRI scanning protocols recommended by the Polish Medical Society of Radiology for the assessment of rheumatic changes within the musculoskeletal system. Sequences considered by the Authors to be obligatory for visualization of inflammatory and destructive lesions within the joints are in bold.

\section{Sacroiliac joints}

- sagittal T2-weighted ${ }^{2}$

- axial ${ }^{b}$ PD FS

- coronal oblique ${ }^{\mathrm{T}}$ TIRM/STIR

- coronal oblique T1-weighted

- coronal oblique T1 FS contrast-enhanced

alncluding the sacroiliac joints for accurate planning of the coronal oblique plane and the assessment of the L/S spine

${ }^{b}$ Axial - a transverse slice of sacroiliac joints perpendicular to the coronal oblique slice

'Coronal oblique - slice of sacroiliac joints in coronal plane relative to the tangent of the posterior surface of S2 vertebral body

\begin{tabular}{l}
\hline \multicolumn{1}{c}{ The spine } \\
\hline - sagittal T1-weighted \\
- sagittal T2 TIRM/STIR \\
- sagittal T2-weighted \\
- axial T2-weighted \\
\hline \multicolumn{1}{c}{ Optional } \\
- coronal T2-weighted \\
- sagittal T1 contrast-enhanced \\
\hline
\end{tabular}

\begin{tabular}{|c|}
\hline The hand ${ }^{\mathrm{a}}$ \\
\hline $\begin{array}{l}\text { - axial T2 FS or PD FS or T2 TIRM/STIR (to be selected } \\
\text { depending on the MRI scanner) } \\
\text { - coronal T1-weighted } \\
\text { - coronal T2 TIRM/STIR or PD FS (to be selected depen- } \\
\text { ding on the MRI scanner) } \\
\text { - coronal T1 FS }\end{array}$ \\
\hline Optional \\
\hline $\begin{array}{l}\text { - coronal T1 FS contrast-enhanced } \\
\text { - axial T1 FS contrast-enhanced } \\
\text { - sagittal PD FS } \\
\text { - dynamic scan: coronal T1-weighted }\end{array}$ \\
\hline
\end{tabular}

\section{The ankle joint and the foot ${ }^{\mathrm{a}}$}

- coronal PD FS or T2 TIRM/STIR

- axial T1-weighted

- axial PD FS or T2 TIRM/STIR

- sagittal PD FS

- coronal PD

- axial T1 FS

\section{Optional}

- axial T1 FS contrast-enhanced

- axial T1 FS contrast-enhanced

- sagittal T1 FS with contrast-enhanced

- dynamic scan: transverse T1-weighted

${ }^{a}$ Coronal slice along the longitudinal body axis axial slice: perpendicular to the longitudinal body axis

\section{The acromioclavicular joint}

- axial T1-weighted

- axial PD FS or TIRM/STIR

- coronal T1-weighted

- coronal PD FS or TIRM/STIR

- sagittal PD FS or TIRM/STIR

- Obligatory: minimum two PD FS or two TIRM/STIR sequences and minimum one T1-weighted sequence from listed above

\section{Optional}

- coronal T1 FS contrast-enhanced

- axial T1 FS contrast-enhanced

- dynamic scan: coronal T1-weighted

\section{The elbow joint}

- axial T1-weighted

- axial PD FS or TIRM/STIR

- coronal PD or TIRM/STIR

- coronal PD FS or TIRM/STIR

- sagittal T1-weighted

- sagittal PD FS

- Obligatory: minimum two PD FS or two TIRM/STIR sequences and minimum one T1-weighted sequence from listed above

\section{Optional}

- axial T1 FS contrast-enhanced

- coronal T1 FS contrast-enhanced

- sagittal T1 FS contrast-enhanced

- dynamic scan: sagittal T1-weighted 


\begin{tabular}{l}
\hline \multicolumn{1}{c}{ The hip joint } \\
\hline - coronal T1-weighted \\
- axial PD FS or STIR/TIRM \\
- coronal PD FS or TIRM/STIR \\
- axial T1-weighted \\
\hline \multicolumn{1}{c}{ Optional } \\
- axial T1 FS contrast-enhanced \\
- coronal T1 FS contrast-enhanced \\
- dynamic scan: coronal T1-weighted \\
\hline
\end{tabular}

\begin{tabular}{l}
\hline \multicolumn{1}{c}{ The knee joint } \\
\hline - axial T1-weighted \\
- axial PD FS \\
- sagittal PD FS or STIR/TIRM \\
- coronal T1-weighted \\
- coronal PD FS or STIR/TIRM \\
\hline \multicolumn{1}{c}{ Optional } \\
- axial T1 FS contrast-enhanced \\
- sagittal T1 FS contrast-enhanced \\
- coronal T1 FS contrast-enhanced \\
- dynamic Scan: transverse T1-weighted \\
\hline
\end{tabular}

\begin{tabular}{l}
\hline \multicolumn{1}{c}{ Temporomandibular joints } \\
\hline \multicolumn{1}{c}{ Assessment of inflammatory lesions: } \\
- axial or coronal T1-weighted \\
- axial T2 STIR/TIRM \\
- coronal T2 STIR/TIRM \\
- coronal T2-weighted \\
\hline Assessment of articular discs and joint mobility: \\
- sagittal oblique PD (articular disc assessment) \\
- sagittal oblique PD FS (mouth open and closed) \\
\hline
\end{tabular}

\section{MRI interpretation}

Subject to the assessment are the T1, T2, PD-weighted and fat saturation or STIR/TIRM sequences.

The assessments are performed in at least two perpendicular planes.

In rheumatology, MRI scans with intravenous administration of contrast agent are performed mainly in order to:

- determine the activity of inflammaion within the synovium;

- determine the activity of erosions;
- confirm the presence of inflammatory lesions if images acquired using other sequences are non-diagnostic;

- make the differential diagnosis (posttraumatic, inflammatory, neoplastic lesions).

Bone marrow edema in rheumatological patients is indicative of osteitis. It is manifested as early as several weeks after the onset of the disease and is considered a very early imaging marker of inflammation. Bone marrow edema is correlated with clinical activity of the disease and elevated levels of acute phase inflammation markers, i.e. erythrocyte sedimentation rate and C-reactive protein. At the early phase of the disease, bone marrow edema is reversible and may resolve under treatment. Bone marrow edema is a radiological symptom preceding the development of erosions.

\section{MRI of sacroiliac joints}

In the diagnosis of early sacroiliitis, MRI scans are evaluated for active and chronic inflammatory changes (Table IV). The MRI-based diagnosis of early sacroiliitis requires detection of bone marrow edema. According to ASAS criteria, the remaining active inflammatory changes, such as synovitis, capsulitis and enthesitis are suggestive of sacroiliitis on the condition of concomitant bone marrow edema in the adjacent areas [9].

MRI scan report should contain a description of active and chronic inflammatory changes, indicate (if possible) the number and location of these changes for diagnostic reasons, and for monitoring of treatment.

Active inflammatory lesions in MRI scans:

- Bone marrow edema (BME) - bone marrow edema is visible as an area of hyperintense signal on T2-weighted images, STIR/TIRM sequence and following contrast administration, and as a low signal area in T1-weighted images. In sacroiliitis, BME is located subchondrally in the iliac or sacral bone; it may accompany bone erosions.

- Synovitis - synovitis is seen as a thickening of synovium and hyperintense signal on T2-weighted, STIR/ TIRM and contrastenhanced T1-weighted FS sequences; it is often concomitant to effusion.

- Capsulitis - capsulitis is seen as a thickening of the sacroiliac joint capsule and hyperintense signal on T2-weighted, STIR/TIRM and contrast-enhanced

Table IV. Types of typical MRI lesions of the sacroiliac joints according to ASAS [9]

\begin{tabular}{|ll|}
\hline \multicolumn{1}{|c|}{ Active inflammatory lesions } & \multicolumn{1}{c|}{ Chronic inflammatory lesions } \\
\hline - Bone marrow edema & -Subchondral sclerosis \\
- Capsulitis & - Erosions \\
- Synovitis & - Fat deposition \\
- Enthesitis & - Bony bridges, ankylosis \\
\hline
\end{tabular}


T1-weighted FS sequences; it is often concomitant to effusion.

- Enthesitis - enthesitis is seen as hyperintense signal of sacroiliac ligaments or capsule entheses on STIR/ TIRM or T1 FS contrast-enhanced images. Features of bone marrow edema are usually detected in the bony part of the enthesis.

\section{Chronic inflammatory changes in MRI scans}

1. Subchondral sclerosis of the sacroiliac joint: low-intensity signal in all sequences.

2. Erosions: bony defects at the joint margin, including active erosions (i.e. filled with inflammatory tissue of low signal intensity on T1-weighted images without contrast enhancement and high signal intensity on contrast enhanced T1-weighted, PD FS, and STIR/ TIRM images) and inactive erosions (no abnormal enhancement following contrast agent administration).

3. Subchondral fatty conversion of bone marrow: hyperintense signal on $\mathrm{T} 1, \mathrm{~T} 2$, and PD-weighted images suppressed in fat-saturation sequences, no abnormal enhancement following contrast agent administration.

4. Ankylosis, or bony union across the joint.

\section{MRI of the spine}

In rheumatoid patients, MRI of the spine is usually performed in order to assess the atlantooccipital region (synovial pathologies, odontoid erosions and inflammatory cysts, C1/C2 subluxation, C2 disc herniation) and to search for inflammatory lesions in the course of axial spondyloarthropathies.

Typical for axial SpA findings include inflamed vertebral bodies, including bone marrow edema in the anterior parts of the endplates (shiny corners or corner inflammatory lesions, CIL) as a potential site for the development of erosions or syndesmophytes in the course of ankylosing spondylitis, as well as intervertebral discs, intervertebral joints and costovertebral joints inflammation.

In addition, areas of fatty marrow conversion, syndesmophytes, erosions, and ankylosis are also visible in the scans. Interpretation of the images is similar to that of sacroiliitis.

\section{MRI of peripheral joints}

Inflammatory lesions in joint cavities, tendon sheaths and bursae: thickening of the synovium, enhancement of inflamed synovium, effusion, erosions, inflammatory cysts (geodes), ligaments and tendons damage.

Synovitis is assessed on T1-weighted fat-saturation sequences following contrast administration. The enhancement within the synovial membrane should be examined within no longer than 5-10 minutes from contrast administration. After this time, the contrast agent permeates into the synovial fluid. Fibrosis within the synovium is the subject to low contrast enhancement.

Bone marrow edema in the course of the inflammation is seen as hyperintense areas on T2, PD-weighted images, best visible in fat-saturated or STIR/TIRM sequences, hypointense on T1-weighted images, with enhancement following contrast agent administration.

Inflammatory cysts are inflammatory focal lesions within the bone marrow. They are more limited than the areas of bone marrow edema.

Erosions are trabecular bone defects with not preserved cortical bone continuity.

\section{Other changes detectable in MRI of the musculoskeletal system in rheumatological patients}

- Tendinitis secondary to tenosynovitis, tendon damage;

- Inflammatory lesions of subdermal tissue in the course of scleroderma [11];

- Dermatomyositis, polymyositis (muscles inflammation, biopsy site identification);

- Deficiency-related fractures (sacral bone, femoral neck, metaphyses, trabecular bone);

- Avascular necrosis;

- Neuropathies;

- Rheumatoid nodules;

- Polymyalgia rheumatica (identification of inflammation).

MRI assessment is usually qualitative. Examples of semiquantitative assessment of inflammatory lesions in the carpal and metacarpophalangeal joints in RA include: RAMRIS (Rheumatoid Arthritis MRI Scoring System) [12], PsAMRIS (Psoriatic Arthritis MRI Scoring System) [13], or the Canadian scoring system for sacroiliac joint inflammation [14].

The RAMRIS system includes definitions of basic RA symptoms at individual disease stages, descriptions of MRI scanning sequences and semi-quantitative assessment of the inflammatory-destructive lesions range within the carpal and metacarpophalangeal joints $[15,16]$. The use of RAMRIS is an attempt to standardize the assessment of the severity and the monitoring of the treatment of inflammatory lesions in carpal joints.

According to RAMRIS assessment:

- The intensity of synovitis is assessed in three areas of the wrist: distal radioulnar joint; radiocarpal joint; metacarpal and carpometacarpal joints. 
Synovitis is assessed in a staging scale from 0 to 3 that describes the volume of enhanced synovium following contrast agent administration:

- Stage 0 - no features of synovitis;

- Stage 1 - mild synovitis (up to $1 / 3$ of joint volume);

- Stage 2 - moderate synovitis (1/3 to 2/3 of joint volume);

- Stage 3 - severe synovitis (more than 2/3 of joint volume);

- Bone marrow edema (BME) is assessed separately for each carpal bone, distal radial epiphysis, the ulna, base parts of metacarpal bones $\|-\mathrm{V}$, and metacarpophalangeal joints bones. The stages of bone marrow edema (from 0 to 3) reflect the percentage volume of bone affected by BME.

- Grade 0 - no bone marrow edema;

- Grade 1 - BME affecting up to 1/3 of the assessed bone volume;

- Grade 2 - BME affecting 1/3 to 2/3 of the assessed bone volume;

- Grade 3 - BME affecting more than 2/3 of the assessed bone volume.

- Bone destruction grade (erosion size) is assessed separately for each of the aforementioned bones in a scale of $0-10$ reflecting the percentage volume of bone being destroyed due to erosion.

Grade 0 - normal bone (no erosions). The successive grades 1-10 reflect the degree of bone destruction in $10 \%$ increments (e.g. grade 1 - up to $10 \%$ of bone volume being destroyed due to erosion; grade 2 from $11 \%$ to $20 \%$ of bone volume being destroyed and so on) [16].

In addition, a very promising method of dynamic contrastenhanced MRI (DCE-MRI) for the assessment of the degree of enhancement of inflamed synovium has been developed in recent years. The examination consists of imaging of the same slices at intervals of several seconds, before and immediately after intravenous contrast administration, over a period of ca. 10 minutes. It allows evaluation of the enhancement degree in the area of inflamed synovium. Measurements obtained from the analysis reflect synovial perfusion and permeability of capillary vessels within the inflamed tissue. They are correlated with the degree of synovial vascularization which may be particularly useful in monitoring of treatment $[17,18]$.

\section{The role of MRI in treatment of rheumatic diseases}

Detection of inflammatory lesions in the sacroiliac joints on MRI scans is often crucial for making a diagnosis of axial spondyloarthropathy, particularly in the preradiographic stage (before definite lesions are detected on radiographs). Early diagnosis and treatment can con- tribute to a better chance of remission, particularly as certain biological agents are documented to be of most efficacy in the preradiographic stage of axial spondyloarthropathy [19].

The authors declare no conflict of interest.

\section{References}

1. Schueller-Weidekamm C, Mascarenhas VV, Sudol-Szopinska I, et al. Imaging and interpretation of axial spondylarthritis: The radiologist's perspective-consensus of the arthritis subcommittee of the ESSR. Semin Musculoskelet Radiol 2014; 18 : 265-279.

2. Rudwaleit $M$, van der Heijde $D$, Landewé $R$, et al. The development of Assessment of SpondyloArthritis international Society classification criteria for axial spondyloarthritis (part II): validation and final selection. Ann Rheum Dis 2009; 68: 777783.

3. Rudwaleit M, van der Heijde D, Landewé R, et al. The Assessment of SpondyloArthritis International Society classification criteria for peripheral spondyloarthritis and for spondyloarthritis in general. Ann Rheum Dis 2011; 70: 25-31.

4. Sudol-Szopinska I, Urbanik A. Diagnostic imaging of sacroiliac joints and the spine in the course of spondyloarthropathies. Pol J Radiol 2013; 78: 43-49.

5. Van den Berg R, van der Heijde DMFM. How should we diagnose spondyloarthritis according to the ASAS classification criteria: a guide for practicing physicians. Pol Arch Med Wewn 2010; 120: 452-457.

6. Filipowicz-Sosnowska A, Zimmermann-Górska L. Choroby układowe tkanki łącznej. In: Choroby wewnętrzne. Szczeklik A (ed.). Stan wiedzy na rok 2011. Medycyna Praktyczna 2011.

7. Mandl P, Navarro-Compán V, Terslev L, Aegerter P. EULAR recommendations fort the use of imaging in spondyloarthritis in clinical practice. Ann Rheum Dis 2014; 73 (Suppl. 2): 427-428.

8. Colebatch AN, Edwards CJ, Østergaard M, et al. EULAR recommendations for the use of imaging of the joints in the clinical management of rheumatoid arthritis. Ann Rheum Dis 2013; 72: 804-814.

9. Sieper J, Rudwaleit M, Baraliakos X, et al. The Assessment of SpondyloArthritis international Society (ASAS) handbook: a guide to assess spondyloarthritis. Ann Rheum Dis 2009; 68 (Suppl. 2): ii1-ii44.

10. Nistala K, Babar J, Johnson K, et al. Clinical assessment and core outcome variables are poor predictors of hip arthritis diagnosed by MRI in juvenile idiopathic arthritis. Rheumatology 2007; 46: 699-702.

11. Schanz S, Fierlbeck G, Ulmer A, et al. Localized Scleroderma: MR Findings and Clinical Features. Radiology 2011; 260: 817-824.

12. Østergaard M. An introduction to the EULAR-OMERACT rheumatoid arthritis $M R I$ reference image atlas. Ann Rheum Dis 2005; 64 (Suppl. 1): i3-7.

13. Østergaard M, McQueen F, Wiell C, et al. The OMERACT Psoriatic Arthritis Magnetic Resonance Imaging Scoring System (PsAMRIS): Definitions of key pathologies, suggested MRI 
sequences, and preliminary scoring system for PsA hands. J Rheumatol 2009; 36: 1816-1824.

14. Maksymowych WP, Inman RD, Salonen D, et al. Spondyloarthritis Research Consortium of Canada magnetic resonance imaging index for assessment of sacroiliac joint inflammation in ankylosing spondylitis. Arthritis Care Res 2005; 53: 703-709.

15. Ejbjerg B. The EULAR-OMERACT rheumatoid arthritis MRI reference image atlas: the wrist joint. Ann Rheum Dis 2005; 64 (Suppl. 1): i23-47.

16. Kapuścińska K, Urbanik A, Wojciechowski W. Standardisation of the MRI image evaluation in the diagnostics of rheumatoid arthritis within the wrist and metacarpophalangeal joints. Pol Jadiol 2009; 74: 77-82

17. Wojciechowski W, Czaplicka K, Włodarczyk J, Tabor Z. Correlation between manual 0.2T MRI assessment of synovitis and EULAR-OMERACT scores of the wrist in patients with rheumatoid arthritis. J Magn Reson Imaging 2014; 39: 1171-1177.

18. Wojciechowski W, Tabor Z, Urbanik A. Assessing synovitis based on dynamic gadolinium-enhanced MRI and EULAROMERACT scores of the wrist in patients with rheumatoid arthritis. Clin Exp Rheumatol 2013; 31: 850-856.

19. Callhoff J, Sieper J, Weiß A, et al. Efficacy of TNF $\alpha$ blockers in patients with ankylosing spondylitis and non-radiographic axial spondyloarthritis: a meta-analysis. Ann Rheum Dis 2015; 74: 1241-1248. 